\title{
DISCUSIONES
}

CRfTICA, Revista Hispanoamericana de Filosofia

Vol. XXII, No. 66 (diciembre 1990): 115-129

\section{SOBRE CAUSACIÓN Y UNIFICACIÓN SEGÚN WESLEY SALMON*}

LEÓN OLIVÉ

Instituto de Investigaciones Filusóficas, UNAM

Wesley Salmon, en su artículo "Scientific Explanation: Causation and Unification"l señala con gran claridad que en filosofía, tanto como en las ciencias mismas -y me atrevería a decir que en muchas otras disciplinas intelectuales - hay periodos cuando se alcanzan consensos en relación con problemas específicos, luego tiempos en los que se rompen esos consensos, periodos de disensión y de controversia, y a veces tiempos en los que se reconstruyen los consensos, aunque seguramente sobre nuevas bases y bajo diferentes presupuestos.

De la misma manera en la que ahora se reconoce que la formación y ruptura de consensos en la ciencia es un tema de primera importancia que debe investigarse, y que esos procesos deben entenderse y explicarse desde una perspectiva filosófica

* Este trabajo se basa en una discusión previa presentada en el X Simposio de Filosofía sobre "Leyes y explicación", organizado por el Instituto de Investigaciones Filosóficas de la UNAM en agosto de 1990 en la Ciudad de México. En el mismo simposio, Wesley Salmon presentó una primera versión del arlículo que aquí se comenta. Agradezco a Ana Rosa Pérez Ransanz sus comentarios a la versión previa de esta discusión.

1 Publicado en este número de Crítica, pp. 3-21. Al mismo número corresponden las citas que damos traducidas más adelante. 
y también una sociológica, de ese mismo modo debería reconocerse que la formación y ruptura de consensos en filosofía es un problema que también requiere comprensión y explicación.

El trabajo de Salmon, especialmente su impresionante libro Cuatro décadas de explicación científica (1989), muestra claramente que ha habido a lo largo del tiempo un cambio importante en las concepciones filosóficas acenca de lo que de hecho es la explicación científica y acerca de lo que debería ser. Este cambio está relacionado con el desarrollo de las diversas áreas de la filosofía, así como con el de la ciencia misma y con la evolución de las diferentes concepciones científicas acerca del mundo.

Tal vez tomar conciencia de la evolución de la discusión filosófica y de su conexión con el desarrollo de los diversos campos de la filosofía, así como con el desarrollo de las mismas ciencias, sea una de las lecciones más importantes que debemos aprender de un análisis filosófico de la noción de explicación científica que esté bien informado históricamente, como el que Salmon nos ha ofrecido en su libro Cuatro décadas de explicación científica, y que ha resumido en el artículo citado y que a continuación discutiremos.

La importancia de estas ideas reside en que, en efecto, si tomamos conciencia de los cambios en las concepciones filośficas de la explicación científica, podemos entender la sugerencia de Salmon de que "no parece plausible esperar una exitosa caracterización de la explicación científica en términos de algún esquema formal simple o de una simple formulación lingüística" (p. 20). Y más aún, quizá esta perspectiva realmente pueda ofrecer razones para creer que la situación es como el propio Salmon lo sugiere siguiendo a su estudiante Gemes (véase p. 20), a saber, que las teorias científicas -y podríamos agregar que para el caso también las visiones del mundo- bien pueden tener diferentes virtudes explicactivas, y nosotros debemos evaluar las teorías en términos de esas virtudes explicativas. 
En lo que sigue haré algunos comentarios acerca de la preocupación central de Salmon en el artículo antes mencionado, a saber, el intento de considerar la posibilidad de que la concepción de la unificación y la concepción causal "sean realmente compatibles y complementarias" (p. 11).

Salmon reconoce explícitamente que la idea de que la explicación consiste principalmente en la exposición de los mecanismos subyacentes, la concepción causal, "involucra conseguir un conocimiento de la manera en que funcionan las cosas. . lo que queremos hacer — nos dice Salmon-es abrir la caja negra y sacar a la luz sus mecanismos internos" (p. 18). Esto es claramente ponerse del lado del realismo en la disputa realismo/anti-realismo, como Salmon claramente lo admite en su trabajo y lo ha defendido explícitamente en otras obras, por ejemplo en Cuatro décadas de explicación científica.

Salmon ve a las dos concepciones — la de la unificación y la causal- como complementarias, lo cual implica que las dos deberían ser aceptadas como buenas, reconociendo que ninguna de las dos es completa por sí misma para dar cuenta de la explicación científica. Ahora bien, la aceptación de la concepción causal implica la aceptación de sus compromisos realistas. Pero debemos dejar claro que una concepción realista de la ciencia no se sigue de la concepción causal, sino que está presupuesta por ésta. La concepción causal presupone una concepción realista de la ciencia.

Por otra parte, la concepción de la unificación parece ser compatible tanto con concepciones realistas como con antirealistas. Pero vale la pena observar que por lo menos en la formulación original de Friedman en 1974, parece tenerse en mente más una concepción empirista de la ciencia que una concepción realista: “... ésta es la esencia de la explicación científica - la ciencia aumenta nuestra comprensión del mundo por medio de la reducción del número total de fenómenos 
independientes que tenemos que aceptar como últimos, o como dados. Un mundo con menos fenómenos independientes es, si todo lo demás se mantiene igual, más comprensible que uno con más (fenómenos independientes)" (Friedman 1974, p. 15).

Kitcher, en una de las más recientes y serias formulaciones de la unificación, publicada en 1989, reconoce que él quiere evitar compromisos realistas, y de hecho sugiere que evita una discusión metafísica, pero me parece que lo que hace es inclinarse también por una concepción empirista de la ciencia. Kitcher resume su posición como sigue: "explicar es acomodar el fenómeno en una imagen ('picture') unificada tanto como podamos. Lo que aparece en los límites de este proceso es ni más ni menos que la estructura causal del mundo" (p. 500). Previamente, Kitcher elabora su punto de vista, sobre la línea trazada por Friedman: "La ciencia hace avanzar nuestra comprensión de la naturaleza mostrándonos cómo derivar descripciones de muchos fenómenos, utilizando los mismos patrones de derivación una y otra vez, y al demostrar esto, nos enseña cómo reducir el número de tipos de hechos que tenemos que aceptar como últimos (o como brutos)" (p. 432). Me parecen claras las connotaciones empiristas de este punto de vista.

Sea como sea, afirmar que la concepción de la unificación y a la causal son compatibles y complementarias implica la aceptación de los compromisos realistas de la segunda, de la causal. Veamos cuáles son los problemas que surgen de esta idea.

En primer lugar, hasta donde puedo comprender la concepción de Railton (1981) de un texto explicativo ideal, esta noción parecería también presentarse como neutral con respecto a la disputa realista/anti-realista. No es claro para mí hasta qué punto Salmon acepta esta noción de un texto explicativo ideal; sin embargo, lo que dice en la página 16 de su artículo parece sugerir que sí la acepta.

Ahora bien, el problema es que hablar sobre un texto explicativo ideal, expresado en los términos realistas que Sal- 
mon admite explícitamente, parece conducir a la idea de que existe una única, completa y verdadera teoría del mundo, lo que Putnam ha caracterizado como una tesis central del llamado realismo metafísico (véase Putnam, 1981). Creo que efectivamente se llega a esta idea, pues recordemos que de acuerdo con Railton lo que nos ofrecen las porciones o aspectos del texto ideal es lo que él llama información explicativa. De esto se sigue que uno de los objetivos importantes de la ciencia debería ser el procurar tantas y tan completas piezas de información explicativa como sean posibles. Y en la medida en que las ciencias alcancen esta meta, se acercarán más al texto explicativo ideal, es decir, nos ofrecerán historias más cercanas a la verdad. La verdad es lo que estaría incorporado en el texto explicativo ideal.

Creo que esto es también lo que Laudan (1981) ha llamado (y criticado como) el "realismo convergente". Otra manera de plantear el problema es decir que si la idea de un texto explicativo ideal se introduce cuando las dos concepciones se ponen juntas y se ven como complentarias, puesto que la concepción causal presupone una concepción realista, entonces se cae en un compromiso con el realismo convergente.

He sostenido en otros trabajos y en otras ocasiones (1984a, 1987,1988 ) que el realismo metafísico y el realismo convergente son insostenibles. Una de las razones principales para rechazar esta concepción es que la idea de una teoría única, completa y verdadera acerca del mundo lleva a concepciones metafísicas indeseables. Por ejemplo, para decirlo con las palabras de Putnam, a la idea de que existen objetos que son autoidentificantes y que son independientes de la mente, lo cual a la vez implica — a mi modo de ver- una identificación de la razón con la realidad.

La cuestión acerca de cuáles son los objetos que hay en el mundo no tiene sentido a menos que se plantee en relación con algún marco conceptual. La idea de objetos autoidentificantes es absurda puesto que los objetos obviamentes son identifica- 
dos siempre desde algún punto de vista. La gente usa marcos conceptuales para identificar objetos y los objetos son identificados sólo por medio de los marcos conceptuales. La noción de autoidentificación implica la idea de que los objetos tienen conceptos construidos dentro de ellos mismos, de manera que parece surgir una relación indisoluble entre los objetos y su interpretación, entre la realidad y las ideas. La única, completa y verdadera descripción de la realidad, o el texto explicativo ideal, debería ser aquél que se expresara en los términos de aquellos conceptos que en la realidad están relacionados con los objetos. A mi modo de ver ésta es una consecuencia indeseable de la concepción realista metafísica, pues no deja lugar para una divergencia cultural y conceptual, es decir para que existan diferentes concepciones acerca del mundo, aunque todas ellas puedan considerarse correctas - siempre y cuando, por supuesto, tales concepciones no sean contradictorias. Me parece que dejar espacio para esta idea es un requisito de una correcta comprensión del desarrollo y de la evolución de la ciencia.

También he defendido en otras ocasiones (1988) la idea de que una concepción realista de la ciencia debe reconocer la existencia de una realidad no contaminada por una conceptualización, sin embargo debe abandonar la idea de que existe una única teoría completa y verdadera acerca del mundo. Debe reconocerse la existencia de diversos marcos conceptuales, los cuales, a pesar de ser diferentes, pueden ofrecer concepciones correctas de la realidad; esto es decir que son posibles diversas y diferentes descripciones correctas de la realidad, incluyendo de los mecanismos responsables de la ocurrencia de los sucesos. Existe un sentido en el que podemos reconocer la convergencia, y es el sentido de que los mecanismos reales responsables de la ocurrencia de los sucesos se describan de maneras más precisas, y exista así una cadena de modelos anidados, de los más burdos a los más finos y precisos. Pero esto no implica 
una convergencia hacia una única teoría del mundo, completa y verdadera.

Salmon señala correctamente que uno de los rasgos de la explicación científica es su contribución a nuestra comprensión del mundo. Señala también correctamente que esta comprensión necesariamente presupone "una visión general del mundo -una Weltanschauung. La comprensión de los fenómenos del mundo requiere que sean acomodados dentro de esta visión general del mundo" (p. 17).

La introducción de la noción de visión del mundo, de Weltanschauung, casi de manera inmediata llama al fantasma del relativismo. Este es un riesgo del cual Salmon está perfectamente conciente y por eso defiende la idea de que una comprensión científica, o una visión científica del mundo, es superior a otras, digamos míticas o religiosas. "La superioridad de la comprensión científica - dice Salmon- reside en el hecho de que tenemos mucho mejores razones para pensar que es verdadera - aunque tal vez algunas otras visiones del mundo pudieran tener mayor atractivo psicológico" (pp. 17-18).

El problema con esta respuesta es que las razones para creer que una idea es verdadera son intrínsecas a la misma visión del mundo, al mismo marco conceptual, en el que la idea se formula. Y esto ocurre no ś́lo con respecto a las razones, sino también con respecto a los criterios para decidir qué es lo que cuenta como una razón, y más aún como una buena razón. Un visión del mundo religiosa, por ejemplo, contará a la revelación como una buena razón para creer, y más aún, lo revelado ciertamente se considerará como verdadero; pero supongo que una visión científica del mundo rechazará esto como criterio para aceptar creencias. De manera que no podemos tan fácilmente como pretende Salmon evitar el riesgo de relativismo y suponer que la comprensión científica es directamente superior a cualquier otra forma de comprensión.

Quisiera sugerir, sin embargo, que la línea de argumenta- 
ción de Salmon es correcta siempre y cuando se reconozca el compromiso previo con una concepción realista de la ciencia.

Esto puede apreciarse si vemos que lo que Salmon está sugiriendo es que la concepción de la unificación es correcta siempre y cuando las explicaciones contribuyan a lograr una comprensión científica del mundo, lo cual a la vez significa "una comprensión en términos de un acomodo de los fenómenos en una visión del mundo que sea científica", que ya sea científica (véase p. 17). De manera que el intento de ver a los dos enfoques como complementarios y compatibles requiere una previa caracterización de lo que es la comprensión científica, tanto como una articulación y defensa previa de una concepción realista de la ciencia.

Veamos el problema de la comprensión científica. Creo que la pretensión de que la comprensión es lo que los científicos de hecho creen y lo que nos hacen creer, sería muy simplista y no ayudaría para nada.

Para tener una mejor elucidación de la comprensión científica, debemos tener una idea que provenga del conccimiento previamente adquirido, y de nuestras concepciones metafísicas - acordes con dicho conocimiento- acerca de cómo es en general el mundo. De acuerdo con el conocimiento científico que tenemos hoy en día y según lo que dicen muchos campos de la ciencia, creo que es posible esbozar una respuesta a la pregunta acenca de lo que debe ser una adecuada comprensión científica del mundo, y esta respuesta está relacionada con la forma en la que se entienden actualmente los objetivos de la ciencia.

Para presentar más claramente la idea, quisiera recordar una crítica que he hecho al empirismo constructivista de van Fraassen (Olivé 1984b), a saber, que él está dando cuenta del conocimiento tecnológico pero no del genuino conocimiento científico, en la medida en la que considera que el objetivo de la ciencia es salvar las apariencias. Salvar las apariencias resulta de interés para los seres humanos en la medida en la que eso permita predicciones exitosas y sea posible así manipular 
los fenómenos. Creo que esta manipulación de los fenómenos es algo característico de la tecnología más que de la ciencia.

Me parece digno de enfatizar este punto para fortalecer la idea de que lo que es peculiar, lo que otorga el carácter peculiar de los marcos conceptuales científicos, o de las concepciones del mundo científicas, reside en que realmente consiguen un conocimiento de la manera en que funcionan o trabajan las cosas, y esto lo hacen describiendo los mecanismos que son responsables de la ocurrencia de los fenómenos.

Por supuesto no quiero decir con esto que algunas partes de las ciencias, o de las concepciones teóricas de las ciencias - teorías, modelos, etc. - que no ofrecen o que no han ofrecido una descripción de los mecanismos subyacentes no deban considerarse como partes de la ciencia. Por ejemplo, no sugiero que neguemos que la biología ha sido una ciencia durante todo el tiempo en que las teorías evolutivas se desarrollaron sin ser capaces de señalar los mecanismos responsables de las variaciones, mecanismos que hoy en día son descritos por la genética y la biología molecular. Pero en esta discusión hay un punto muy importante en relación con la idea de los objetivos de la ciencia. Una cosa es - y es correcta - reconocer cuáles han sido de hecho los objetivos que las ciencias han tenido en el pasado, y evaluar la medida en que las ciencias han logrado cumplir esos objetivos. Pero una cosa muy distinta es pretender que lo único que puede decirse de la explicación científica es una descripción de lo que de hecho ofrecen como explicación las teorías que han sobrevivido hasta la fecha.

Con esto entramos a una discusión acerca del papel.normativo o del papel descriptivo de la filosofía de la ciencia en relación con las ciencias mismas y las actividades científicas. Ha existido una larga tradición que ve como opuestos al papel descriptivo y al papel normativo de la epistemología y de la filosofía de la ciencia. Creo que esta oposición ha estado detrás del trabajo de Salmon, sin que explícitamente haya sido traída al tapete de la discusión. 
Quisiera sugerir que esta dicotomía debe tomarse en cuenta, pero no debe verse como un par de opuestos, sino al contrario, como una par de características que son complementarias y que deben estar presentes en cualquier filosofía de la ciencia satisfactoria. No hay una oposición entre descripción y prescripción, de la misma manera que en la sugerencia de Salmon no hay una oposición entre una concepción de la unificación y una causal.

Esto significa sugerir que un análisis filosófico de la explicación científica ciertamente debe describir la manera en la cual diferentes teorías científicas, en diferentes etapas de su desarrollo y en diferentes épocas, han explicado los fenómenos que han pretendido explicar. También esta concepción tiene que reconocer que algunas teorías, por ejemplo la mecánica cuántica, obtienen una calificación muy alta con respecto a ciertas virtudes, pero calificaciones muy bajas con respecto a otras virtudes. Debe insistirse que una filosofía de la ciencia tiene también que discutir y a veces que sacar a la luz los estándares y los objetivos de las teorías científicas y de la actividad científica. Aquí es pertinente recordar que algunos objetivos específicos que se plantean en ciertas investigaciones científicas son responsables de la realización de algunos programas de investigación específicos. Por ejemplo mucho del trabajo que se desarrolla hoy en día en electrodinámica estocástica está motivado por el objetivo de ofrecer los mecanismos subyacentes que explicarían fenómenos al nivel cuántico, y así ser capaces de preservar ciertos supuestos metafísicos que son especialmente favorecidos por algunos científicos. Esto quiere decir que este objetivo se persigue porque se tienen ciertos presupuestos metafísicos acerca del mundo, los cuales a la vez corresponden con una concepción realista de la ciencia y con una concepción -que se deriva de la anteriorde la manera en la que deben darse las buenas explicaciones científicas. 
Pero debo advertir que esta posición no sugiere que los metodólogos o los filósofos deban decirles a los científicos cómo hacer su trabajo. Lo que sugiero es que los científicos y las actividades científicas inevitablemente tienen supuestos metafísicos, concepciones del mundo subyacentes, de la misma manera en la que tienen estándares y objetivos relacionados con estas concepciones metafísicas, todo lo cual a la vez condiciona los criterios para decidir lo que debe ser una buena explicación científica. La clase de prescripción acerca de la cual estoy hablando, entonces, se deriva de la propia metodología y de la normatividad interna a las visiones científicas del mundo. No consiste en una recomendación a priori que los filósofos puedan hacer a los científicos, sino más bien se deriva de un análisis global de la ciencia, de sus valores, de sus fines y de sus presupuestos en general, de su evolución y desarrollo, así como de lo que la propia ciencia dice acerca de cómo es el mundo.

Hemos aprendido de la ciencia -aunque tal vez no de la mecánica, pero sí de muchas ramas de la ciencia- que efectivamente hay mecanismos subyacentes que producen fenomenos. Esto ofrece ciertamente una explicación de cierto tipo de fenómenos, nos ofrece también una buena comprensión de cómo es el mundo, y en muchas ocasiones nos permite manipular ese mundo. Es correcto entonces establecer que uno de los objetivos de la ciencia sea el que se ha logrado exitosamente en varios campos científicos. Pero debemos reconocer que muy bien puede ser el caso de que este tipo de explicación no sea posible de obtenerse en todas las ciencias, y no en todos los momentos de su desarrollo. Surge' entonces la necesidad de una discusión en la ciencia particular de que se trate acerca de por qué, o cuáles sonlas razones por las que ese tipo de explicación no es posible, al menos por el momento, en función de los marcos teóricos particulares de esa ciencia y de la tecnología disponible. Puede haber razones en ciencias específicas por las cuales no quede otra posibilidad que aceptar explicacio- 
nes que no satisfagan las condiciones de las explicaciones causales de grano fino de las cuales habla Salmon, pero también puede haber buenas razones para no abandonar la búsqueda de mecanismos subyacentes. En el caso de la mecánica cuántica esta cuestión está lejos de haberse resuelto, y como ya comenté antes, actualmente se siguen programas de investigación de diferentes clases, precisamente porque hay una resistencia a abandonar la idea de mecanismos subyacentes.

En conclusión, quisiera decir que debemos tomar muy en serio la sugerencia de Salmon de que ya no es posible pensar en una formulación simple, o en un simple esquema formal acerca de lo que es o lo que debería ser la explicación científica. La concepción de la unificación puede verse como complementaria a la causal, pero esto supone un compromiso realista previo: lo que hace científica a la comprensión científica del mundo es su descripción de los mecanismos subyacentes reales que son responsables de la ocurrencia de los sucesos.

Esto tiene la consecuencia de que la filosofía de la ciencia debe jugar un fuerte papel normativo en conexión con las ciencias. La mejor comprensión científica del mundo proviene del conocimiento de esos mecanismos. Esto no es incompatible con la concepción de la unificación, siempre y cuando la unificación tenga lugar dentro de lo que ya se considera como concepciones científicas del mundo. Cómo son estas concepciones es algo que debe clarificarse antes de poder decir qué es una buena comprensión científica del mundo y por consiguiente cómo debe darse una buena explicación de aspectos del mundo. Desde mi punto de vista, la concepción de la unificación no ofrece un análisis de aquello que es privativo de las visiones científicas del mundo. El análisis de esta posición es aplicable a muchos modos de comprender y de explicar el mundo que no son científicos. Esto no significa que sus aportaciones no sean de importancia, pero quiere decir que se quedan cortas en cuanto a su pretensión de analizar lo que es distintivo de la explicación científica. 
Kitcher, siguiendo a Railton, ha expresado el problema con toda claridad, y supongo que en esto está de acuerdo Salmon: "las diferencias en los puntos de vista acerca de la explicación científica se conectan con diferencias en metafísica" (1989, p. 499).

Lo que he tratado de sugerir es que la concepción de la unificación — por lo menos en las versiones de Friedman y de Kitcher - parecen estar motivadas por supuestos anti-realistas, lo cual tal vez ellos tratan de disfrazar bajo la idea de evitar compromisos metafísicos. El intento de Salmon de ver los dos enfoques como compatibles y complementarios, por consiguiente, sólo puede desarrollarse a costa de algunos supuestos metafísicos que parecen ser muy apreciados por los unificacionistas. Me sorprendería mucho, entonces, que este intento sea bien recibido por los que defienden la concepción de la unificación. Sería interesante conocer la opinión de Salmon sobre este punto, especialmente en vista del esfuerzo que debería hacerse si es que se quiere lograr un nuevo consenso filosófico. acerca de la explicación científica (suponiendo que sea posible).

Desde mi punto de vista, en parte lo que se requiere es una argumentación convincente a favor de la concepción realista de la ciencia que sea independiente de - es decir que no presuponga- la noción de explicación. Esta argumentación debe ofrecer no sólo buenas razones para creer en la existencia real de las entidades no observacionales, sino que también debe dar cuenta del hecho bien conocido y aceptado hoy en día de que el desarrollo de la ciencia implica cambio conceptual, reemplazo de teorías y hasta de visiones del mundo. ¿Cómo es posible que ocurra este reemplazo de teorías y de visiones del mundo, o de marcos conceptuales, lo cual las más de las veces incluye cambios -a veces totales - de explicaciones, y que todavía podamos pretender que la ciencia nos ofrece un conocimiento verdadero de los mecanismos reales que de hecho son responsables de la ocurrencia de los sucesos del mundo? 
Creo que este es uno de los principales desafíos que tiene que enfrentar una concepción realista de la ciencia, y lo que he sugerido en esta discusión es que este desafío debe enfrentarse con éxito y de manera satisfactoria si hemos de creer que la concepción de la unificación y la concepción causal de la explicación científica pueden realmente desarmollarse de manera que resulten compatibles y complementarias.

\section{REFERENCIAS}

Friedman, Michael (1974), "Explanation and Scientific Understanding", Journal of Philosophy, vol. 71, pp. 5-19.

Kitcher, Philip (1989), "Explanatory Unification and the Causal Structure of the World", en Kitcher y Salmon 1989, pp. 410-505. Kitcher, Philip y Wesley Salmon (1989), Scientific Explanation, Minnesota Studies in the Philosophy of Science, vol. XIII, Minneapolis, University of Minnesota Press.

Laudan, Larry (1981), “A Confutation of Convergent Realism”, Philosophy of Science 48, pp. 19-49.

Olivé, León (1984a), "Sobre el realismo convergente", Crítica, Revista Hispanoamericana de Filosofia, vol. XVI, No. 48, pp. 53-78. - (1984b), "Lógica de la invención o lógica del descubrimiento", Actas del I Simposio Hispano-Mexicano de Filosofía, Filosofia e Historia de la Ciencia, Salamanca, España, octubre de 1984, S. Álvarez, F. Broncano, y M. A. Quintanilla (eds.), Ediciones de la Universidad de Salamanca.

(1987), "Two Conceptions of Truth and Their Relationships to Social Theory", Philosophy of the Social Sciences, vol. 17, No. 3, pp. 313-339.

- (1988), Conocimiento, sociedad y realidad, México, Fondo de Cultura Económica.

Putnam, Hilary (1981), Reason, Truth and History, Cambridge University Press.

Railton, Peter (1981), "Probability, Explanation and Information", Synthese, vol. 48, pp. 233-56.

Salmon, Wesley C., Four Decades of Scientific Explanation, en Kitcher and Salmon (1989), pp. 3-219. 
_- "Scientific Explanation: Causation and Unification", en este mismo número de Crítica, pp. 3-21.

Recibido: 20 octubne 1990 . 